A bone fracture that fails to heal after initial treatment can lead to prolonged disability. Regenerative therapies might help to restart the bone-healing process, getting the people affected back in action. By David Holmes; illustration by Lucy Reading-Ikkanda

\title{
STATE OF THE UNION
}

Although most fractures will heal fully, a small but notable proportion do not. These severe cases - known as non-union bone fractures - can cause long-term disability and pain, and often require surgery to rectify. To determine why non-union bone fracture occurs, it is important to understand how bone healing typically progresses.

\section{SNAP TO IT}

\section{0-2 WEEKS}

Immediately after fracture, blood from ruptured vessels inside the bone starts to

coagulate into a swollen mass called a haematoma. At this early stage, it is crucial that the fragments of bone are guided back into place to ensure that they are correctly aligned and to help stem the loss of blood.

\section{SOFTLY DOES IT} 2-6 WEEKS

A soft callus starts to form, made up of new connective tissue, microscopic blood vessels, cartilage and soft spongy bone.

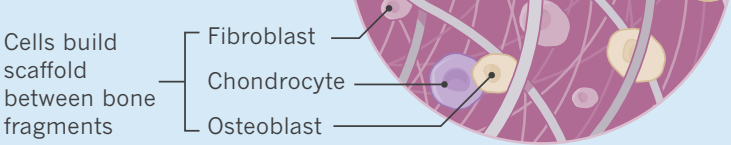

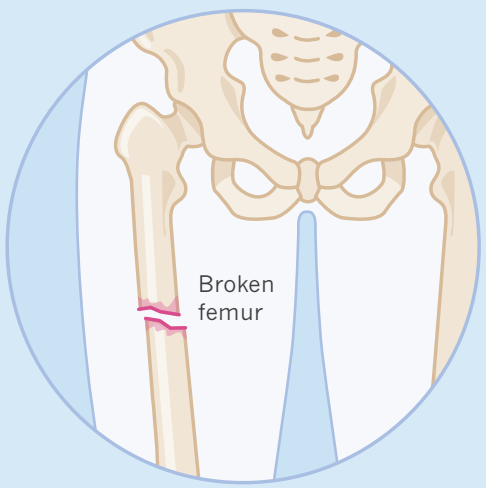

\section{TOUGHENING UP}

\section{WEEKS}

By 12 weeks after fracture, osteoblasts have transformed the soft callus into a hard callus. The length of the healing period depends on the fracture site - bones in the lower limbs can take longer because they are larger.

The healed bone will continue to be remodelled over the coming months and years, in response to stresses imposed by physical activity.
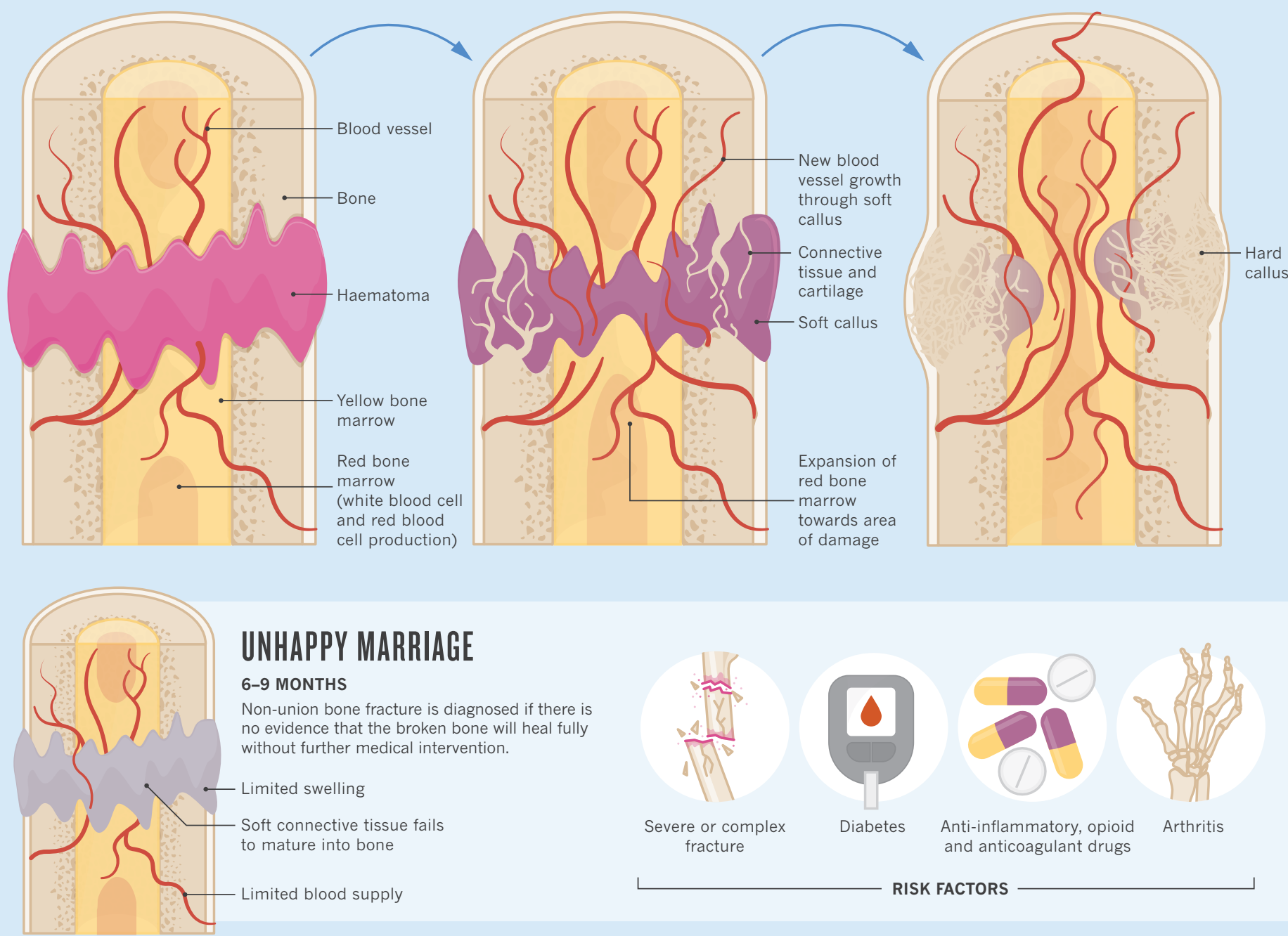

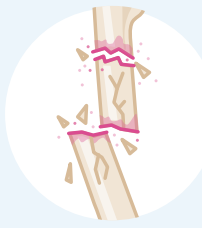

Severe or complex fracture

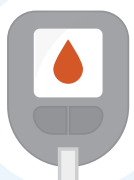

Diabetes

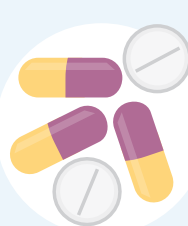

Anti-inflammatory, opioid and anticoagulant drugs

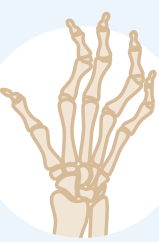

Arthritis 


\section{BREAKING POINT}

Around $5-10 \%$ of bone fractures fail to heal ${ }^{1}$. As the populations of developed countries age, the incidence of fractures, including non-union bone fractures, will increase.

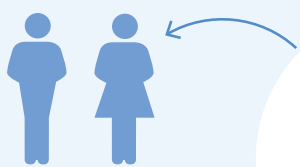

In the United Kingdom, the incidence of fractures ${ }^{2}$ is highest in men aged 15-24 and in women older than 85 .
55

The age beyond

of fracture inen's risk

steadily ${ }^{2}$ - probably

owing to a loss of

bone density.
Almost $8 \mathrm{MILLION}$ fractures are

reported each year in the United States

IIIIIIIIIIIIIIIIIIII IIIIIIIIIIIIIIIIIIIIIIII 400,000 IIIIIIIIIIIIIIIIIIII IIIIIIIIIIIIIIIIIIII

\section{INTO THE BREACH}

Conventionally, the treatment of non-union bone fracture involves surgery to stabilize the fracture site and bone grafting to stimulate the growth of bone and new blood vessels. Researchers are now turning to regenerative medicine to help heal such fractures.

They aim to deliver a type of mononuclear cell called endothelial progenitor cells (EPCs) to the fracture site to induce blood-vessel regeneration and promote bone formation. EPCs express the protein CD34 on their surface, which acts as a marker that enables them to be identified in and extracted from blood.
On days $1-5$ of treatment, the patient is injected with a cytokine that prompts mononuclear cells to migrate from the bone

marrow into the blood.

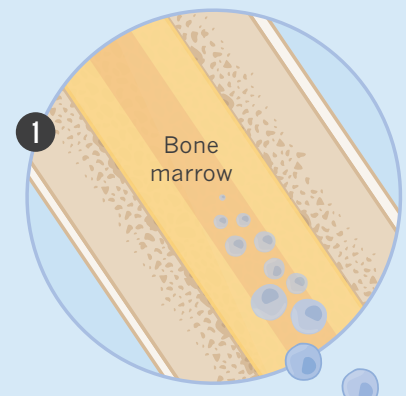

Mononuclear cells are extracted from the patient's blood.

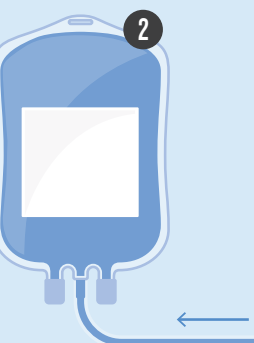

cell

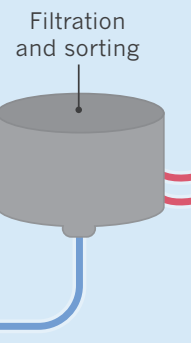

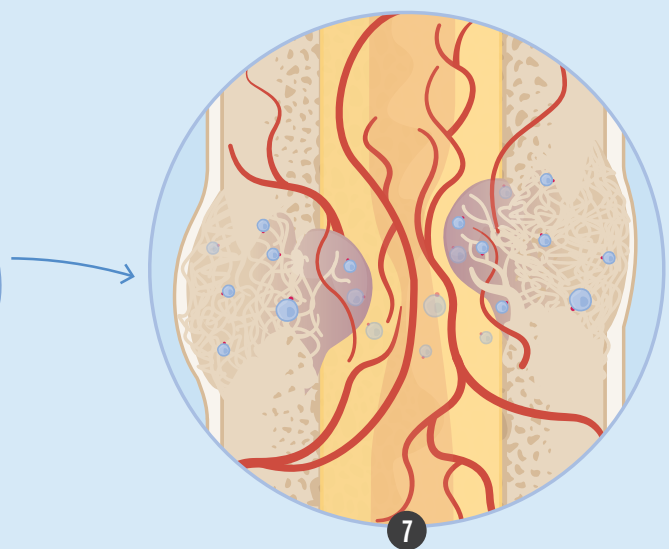

The implanted cells promote blood-vessel growth. These CD34-expressing cells can also transform into other types that can form blood vessels or bone.
Cells expressing CD34 are infused into a gel scaffold.

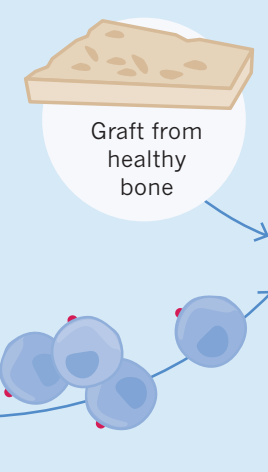

The cell-filled scaffold is implanted at the fracture site, together with a piece of healthy bone.

\section{NEXT STEPS}

A 2014 study ${ }^{4}$ by researchers in Japan showed that treatment with CD34-expressing cells seems to be safe, and bone fractures healed in all people who received the therapy. But the study was too small to determine any clinical benefit. A larger, three-year trial at several institutions to study the effects of CD34-based therapy started in 2015. The results should reveal whether regenerative medicine can help to heal broken bones.

Sources: 1. Mills, L. A. \& Simpson, A. H. R. W. BMJ Open 3, e002276 (2013). 2. Donaldson, L. J., Reckless, I. P., Scholes, S., Mindell, J. S. \& Shelton, N. J. J Epidemiol. Community Health 62, 174-180 (2008). 3. Buza, J. A. III \& Einhorn, T. Clin. Cases Miner. Bone Metab. 13, 101-105 (2016). 4. Kuroda, R. et al. Stem Cells Transl. Med. 3, 128-134 (2014).

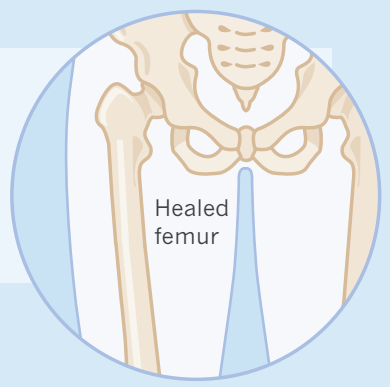

